\title{
TU/e EmonOWEN

\section{3-D Modeling of shielding of magnetic stray fields based on superposition of 2-D models}

\section{Citation for published version (APA):}

Pluk, K. J. W., Jansen, J. W., \& Lomonova, E. A. (2015). 3-D Modeling of shielding of magnetic stray fields based on superposition of 2-D models. IEEE Transactions on Industry Applications, 51(5), 3656-3665. https://doi.org/10.1109/TIA.2015.2422815

DOI:

10.1109/TIA.2015.2422815

Document status and date:

Published: 01/01/2015

\section{Document Version:}

Accepted manuscript including changes made at the peer-review stage

\section{Please check the document version of this publication:}

-A submitted manuscript is the version of the article upon submission and before peer-review. There can be important differences between the submitted version and the official published version of record. People interested in the research are advised to contact the author for the final version of the publication, or visit the $\mathrm{DOI}$ to the publisher's website.

- The final author version and the galley proof are versions of the publication after peer review.

- The final published version features the final layout of the paper including the volume, issue and page numbers.

Link to publication

\section{General rights}

Copyright and moral rights for the publications made accessible in the public portal are retained by the authors and/or other copyright owners and it is a condition of accessing publications that users recognise and abide by the legal requirements associated with these rights.

- Users may download and print one copy of any publication from the public portal for the purpose of private study or research.

- You may not further distribute the material or use it for any profit-making activity or commercial gain

- You may freely distribute the URL identifying the publication in the public portal.

If the publication is distributed under the terms of Article $25 \mathrm{fa}$ of the Dutch Copyright Act, indicated by the "Taverne" license above, please follow below link for the End User Agreement:

www.tue.nl/taverne

Take down policy

If you believe that this document breaches copyright please contact us at:

openaccess@tue.nl

providing details and we will investigate your claim. 


\title{
3-D Modeling of Shielding of Magnetic Stray Fields Based on Superposition of 2-D Models
}

\author{
K.J.W. Pluk, J.W. Jansen and E.A. Lomonova \\ Electromechanics and Power Electronics Group, Department of Electrical Engineering, \\ Eindhoven University of Technology \\ 5612 AZ, Eindhoven, The Netherlands \\ Email: K.J.W.Pluk@tue.nl
}

\begin{abstract}
This paper concerns the superposition of twodimensional semi-analytical models to model three-dimensional shielding configurations. The 2-D semi-analytical models are used to describe the effect of magnetic shielding on the stray field of a single-sided coreless linear permanent magnet motor. The modeling results are compared to 3-D finite element analysis and to measurements. It is found that an accurate description of the tendencies and the order of magnitude is given. Furthermore, the comparison of the superposition of 2-D semi-analytical models with the measurements shows that saturation and neglecting the 3-D effects cause the deviation between model and measurement.
\end{abstract}

Index Terms-Electromagnetic Analysis, Electromagnetic Devices, Finite Element Analysis, Harmonic Analysis, Magnetic Shielding, Mode Matching Methods

\section{INTRODUCTION}

The three-dimensional (3-D) nature of the magnetic fields leads to computationally very intensive 3-D modeling, especially when shielding is applied for the reduction of magnetic stray fields. The possibility to describe the magnetic fields for 3-D shielding configurations by two-dimensional modeling resolves the computational issue. In most electromagnetic devices, this approach is applied as the magnetic fields, used in the desired energy conversion, mainly vary in two of the three dimensions. This implies the application of two-dimensional (2-D) models for describing the full three-dimensional device. With these 2-D models, the resulting magnetic field quantities in two dimensions are obtained by assuming an infinite length in the third dimension. This results in an invariancy in the third dimension of both geometry and field while quantities such as force and flux linkage are obtained for a unit length in this third dimension.

In rotating machines, the validity of 2-D models for 3-D devices is widely covered in literature [1]-[4]. To mimic the effects ignored by assuming a 2-D model, adaptions of the 2-D models are commonly applied, such as, inclusion of endwinding induction in the electrical drive circuit and reduction of the effective stack-length to include the stacking factor. Beside these improvements, based on the motor dimensions, several rules of thumb have been developed to take some 3-D effects into account in the 2-D model [1], [4].

In linear machines, it is also common to use 2-D models to describe the device. For linear machines with a long stroke, it is usual to assume periodicity in the 2-D model, since the device is repeating in one of the modeled dimensions. A 2-D periodic model makes two assumptions that significantly influence their outcome. First of all, it neglects all end-effects in the direction of its periodicity [5]. Secondly, the effects in the third, non-modeled, direction are not taken into account. For some situations, these effects can be compensated in the model by means of an adaption of the material parameters, like the transverse edge effects in linear induction motors [6].

In single-sided linear machines with coreless coils, a large magnetic stray field is present. To reduce the influence of such a linear machine on nearby located magnetic sensitive devices, shielding is applied [7]. Ideally, this magnetic stray field is eliminated by using a solid shield with infinite dimensions, however, due to weight limitations, reluctance forces and cable entries, the shield will be finite in its dimensions and could contain holes. Especially during the design of such a shield, it is important to evaluate many variations of topologies and dimensions. Therefore, during design a fast 2-D model capable of correctly predicting the influences of the shielding is necessary, since such a model greatly reduces the computational efforts.

This paper investigates the validity of a superposition of two-dimensional semi-analytical models, based on Fourier modeling, for three-dimensional shielding configurations. A single-sided coreless linear permanent magnet motor with shielding is considered, which is modeled using both the 2-D semi-analytical model described in this paper and using 3-D finite element analysis. The results of the models are compared for a variation in the $y$-dimensions (the non-modeled dimension in the semi-analytical models) of individual elements in the topology. Measurements are performed for the same topology to verify the forces obtained by the models.

\section{TOPOLOGY}

The topology researched in this paper is based on the Tecnotion UXX ironless series motor [8]. One single stator side together with one, star-connected, coil triplet is considered. Above the coil triplet, a voice coil actuator is located. A shielding plate is located between the coil triplet and the voice coil actuator. The voice coil actuator enables a movement in the vertical direction (the $z$-direction). Only the permanent magnet mover of the voice coil actuator is being modeled, since only the disturbance force on this permanent magnet 


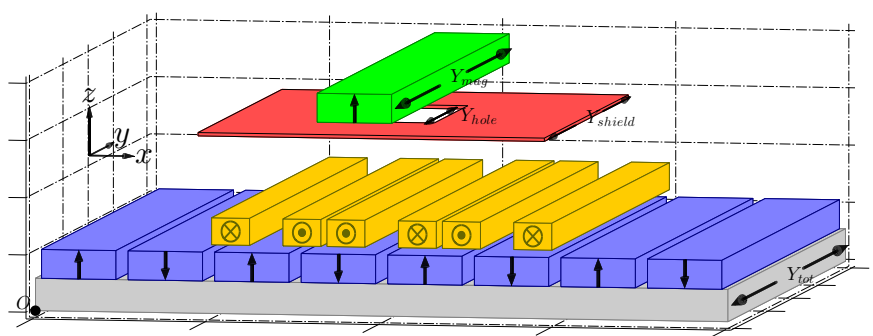

Fig. 1. The topology researched in this paper. The dimensions and specifications of the used materials are given in TABLE I, and $O$ indicates the origin of the coordinate system.

(referred to as victim magnet) is influenced by the shielding and the magnetic stray field. There are reluctance forces present between the victim magnet and the shielding plate, these should be compensated by the coil of the voice coil actuator. Therefore, a rectangular shaped hole is present in the shielding plate to reduce the reluctance forces between the victim magnet and the shield. Furthermore, the shield is centered underneath the victim magnet, which results in a reluctance force purely in the $z$-direction. A 3-D drawing of the full topology is given in Fig. 1. For this topology, three variations are considered in this paper, the $y$-dimension of the victim magnet $Y_{m a g}$, the $y$-dimension of the hole $Y_{\text {hole }}$ and the $y$-dimension of the shield $Y_{\text {shield }}$. These dimensions are indicated in Fig. 1, where also the total $y$-dimension of the topology $Y_{\text {tot }}$ is given.

From the 3-D topology, a cross-section parallel to the $x z$ plane is made in the center of the victim magnet which is used to create the semi-analytical model. For the semianalytical modeling method used, it is necessary to introduce an even periodicity. The width of the period is chosen at $x_{p}=228(\mathrm{~mm})$, which is twice the width of the coil triplet and ensures a minimal influence of the adjacent periods. The dimensions and material properties of the topology are given in TABLE I. The origin is located at the bottom left corner of the back-iron. All permanent magnets have a remanence of $B_{r}=1.23(\mathrm{~T})$ and the magnets in the magnet array are displaced by $28.5(\mathrm{~mm})$ in the $x$-direction, while the position given in the table holds for the first magnet. A DC current source is used to excite the coil triplet. The first phase is excited with a DC current of $5 \cdot 10^{6}\left(\mathrm{~A} / \mathrm{m}^{2}\right)$, due to the starconnection, the returning two phases will carry a DC-current of $-2.5 \cdot 10^{6}\left(\mathrm{~A} / \mathrm{m}^{2}\right)$. The bundles of the coil triplet are geometrical displaced by $38(\mathrm{~mm})$.

\section{2-D SEMI-ANALYTICAL MODEL}

A semi-analytical modeling method based on Fourier analysis [9] is applied to the cross-sectional view of the 3-D topology of Fig. 1. Using a spatial harmonic description, the magnetic flux density in the topology is described. The fundamental frequency, $\omega_{0}$, of the harmonic description is determined by the width of the periodicity and is given by $\omega_{0}=2 \pi / x_{p}$, where $x_{p}=228(\mathrm{~mm})$ represents the width of the periodicity. The choice of this fundamental frequency
TABLE I

THE CENTER COORDINATES, DIMENSIONS AND MATERIAL PROPERTIES OF THE PARTS OF THE TOPOLOGY GIVEN IN FIG. 1.

\begin{tabular}{l|c|c|c|l} 
Model Part & Region & $\begin{array}{c}\text { Position }(\mathrm{mm}) \\
x, y, z\end{array}$ & $\begin{array}{c}\text { Dims }(\mathrm{mm}) \\
x, y, z\end{array}$ & Material \\
\hline Backiron & II & $114,35,4.8$ & $228,70,9.6$ & $\mu_{r}=870$ \\
Magnet array & III & $14.25,35,13.9$ & $24.5,70,8.6$ & $\mu_{r}=1.03$ \\
Coil array + & V & $64.25,35,24.4$ & $12.5,70,8$ & $\mu_{r}=1$ \\
Coil array - & V & $87.75,35,24.4$ & $12.5,70,8$ & $\mu_{r}=1$ \\
Shield & VII,IX & $105,35,50.5$ & $114,50,1$ & $\mu_{r}=2200$ \\
Hole in shield & VIII & $105,35,50.5$ & $25,20,1$ & $\mu_{r}=1$ \\
Victim magnet & XII & $105,35,61.6$ & $24.5,70,8.6$ & $\mu_{r}=1.03$
\end{tabular}

immediately takes the required even periodic boundary conditions into account. For the modeling, the topology has to be divided into regions where each region can only contain one material (see Fig. 2). Only materials with a linear permeability can be taken into account which implicitly results in the assumption that the materials do not saturate.

By extending the Fourier modeling with the mode-matching method [10], it is possible to include regions in the model which are smaller than the full width of the model. These regions have a different fundamental frequency, where half of the period is matched to the width of the region according to $\omega_{0}^{*}=\pi / x_{w}^{*}$, where $\omega_{0}^{*}$ is the fundamental frequency for a region smaller than the periodicity, and where $x_{w}^{*}$ is the width of that region. Using the mode-matching method, the shield is taken into account in the model. Since only one permeability can be used per region, a total of 4 regions is used to model the shield. One region for the left part of the shield (region VII) and one for the right part of the shield (region IX), and the air in between the shield (region VIII) and next to the shield (region $\mathrm{X}$ ) are taken into account by one region each. By applying the mode-matching method, a physically nonexisting boundary condition has to be placed on the edges in the $x$-direction of the shield. Assuming an infinitesimally small piece of infinite permeable material between the shield and the air next to it, the $z$-component of the magnetic flux density is assumed to be zero on these edges. Due to the large difference in permeability of the shield and the air, the errors introduced by the assumed infinite permeable pieces are very limited [11].

Since no unit permeability is present inside the permanent magnets, each permanent magnet should in principle be taken into account in the model as one region, while using a region for the air in between the magnets as well. If the magnets are modeled in such way, the mode-matching method is applied, which will, therefore, result in assuming the physically nonexisting boundary conditions on the edges of the permanent magnets. The assumed boundary conditions will greatly influence the magnetic field, since it forces the magnetic field on the edges of the permanent magnets in the $x$-direction which is perpendicular to the magnetization. Therefore, the permanent magnet array will be modeled as one region, assuming that the full width of the period contains the permanent magnet material, which also reduces the computational efforts in the model. The actual magnets are now modeled using a 


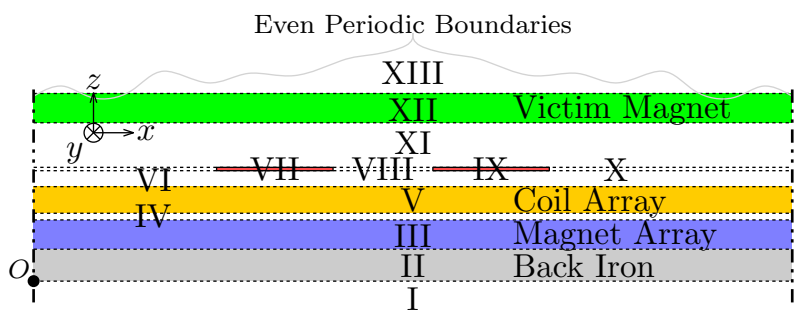

Fig. 2. The division in regions used for the 2-D semi-analytical model.

block shaped magnetization function for which a harmonic description is obtained [7].

The coil triplet is described in an equal manner, the coil (carrying a DC current density of $J=5 \cdot 10^{6} \cos (k \pi / 3)\left(\mathrm{A} / \mathrm{m}^{2}\right)$ for $k=0,1,2)$ is modeled as a block shaped current density function, while an unit permeability is assumed as material. The resulting subdivision in regions is given in Fig. 2.

In each region, the general description based on a summation of $N$ harmonics (the fundamental and its higher order harmonics) gives the magnetic flux density according to the equations given in [11]. The application of this general description in the regions results in a boundary value problem, which is solved by applying boundary conditions on the interfaces between the regions. With the boundary conditions, a solvable set of linear equations is obtained, and therewith, the magnetic flux density in all regions is known. Using the Maxwell stress tensor, the force on the victim magnet is calculated according to the explanations in [12]. The obtained force is given per unit length in the non-modeled direction. This value is multiplied with the $y$-dimension of the model to calculate the actual force for the situation.

\section{Superposition OF 2-D MODELS}

The 2-D semi-analytical model described in the previous section is capable of calculating the force for a topology that is invariant in the non-modeled $y$-dimension. As can be seen in Fig. 1, the topology considered in this paper is not invariant in the $y$-direction. In the topology of Fig. 1, the $y$-dimension of the hole $Y_{\text {hole }}$ and the $y$-dimension of the shield $Y_{\text {shield }}$ are not equal to the $y$-dimension of the victim magnet, coil array, magnet array and back iron.

To include the geometrical variance in the $y$-dimension of the 3-D topology with the 2-D models, a superposition is proposed. The basic principle is to superimpose 2-D models where each of these models describes an invariant part of the 3-D topology. For the topology in Fig. 1, this superposition process is shown in Fig. 3, where the invariant parts are indicated by the dash-dotted lines. Since divisions $A_{1}$ and $A_{2}$ (indicated in the bottom right of Fig. 3) have an equal geometry, these will be combined into a single 2-D model, and the same holds for divisions $B_{1}$ and $B_{2}$. The obtained 2-D models are given in Fig. 4.

Each of the obtained 2-D models is invariant in the $y$ direction. By a summation of the computed quantities of the individual 2-D models, the result of the considered 3-D

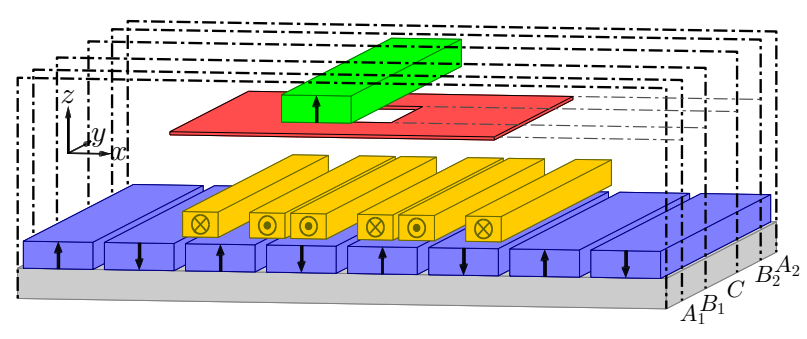

Fig. 3. Division of a 3-D topology in 2-D models with an invariant $y$ dimension.

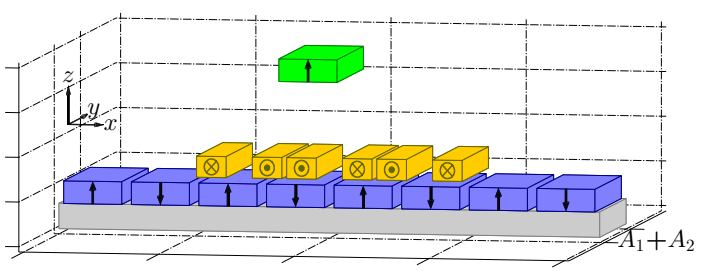

(a)

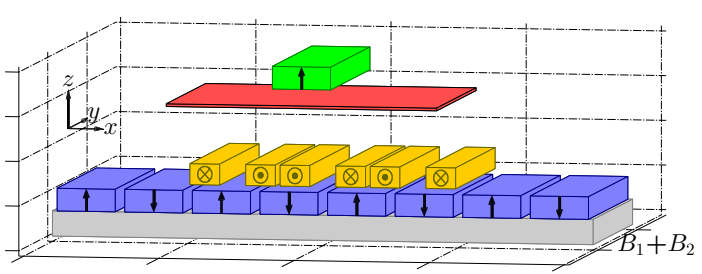

(b)

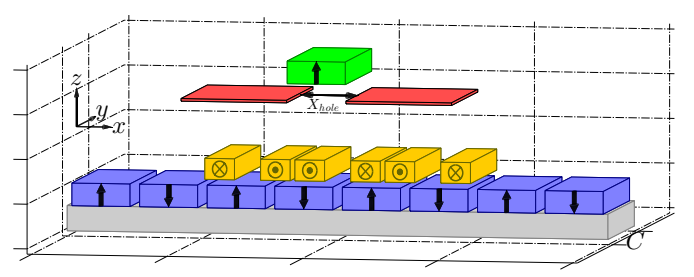

(c)

Fig. 4. The obtained 2-D models for the division illustrated in Fig. 3, where (a) is a 2-D model without a shield, (b) has a full shield (without a hole) and (c) is the model with a shield containing a slit in the $y$-direction.

topology is found. For instance, the force on the victim magnet for this situation is calculated by

$$
F_{\text {tot }}=Y_{C} F_{\text {hole }}+Y_{B} F_{\text {shield }}+Y_{A} F_{\text {no shield }}
$$

where $Y_{A}, Y_{B}$ and $Y_{C}$ are the $y$-dimensions of the invariant models $A, B$ and $C$, respectively, and where $F_{\text {hole }}, F_{\text {shield }}$ and $F_{\text {no shield }}$ are the forces per unit $y$-dimension for the situations with, shield containing a hole, full shield (no hole) and without a shield, respectively. For Fig. 3, $Y_{A}, Y_{B}$ and $Y_{C}$ are given by

$$
\begin{aligned}
& Y_{A}=Y_{\text {mag }}-Y_{\text {shield }} \\
& Y_{B}=Y_{\text {shield }}-Y_{\text {hole }} \\
& Y_{C}=Y_{\text {hole }}
\end{aligned}
$$

where $Y_{\text {mag }}, Y_{\text {shield }}$ and $Y_{\text {hole }}$ are the $y$-dimensions of the victim magnet, shield and hole, respectively. 


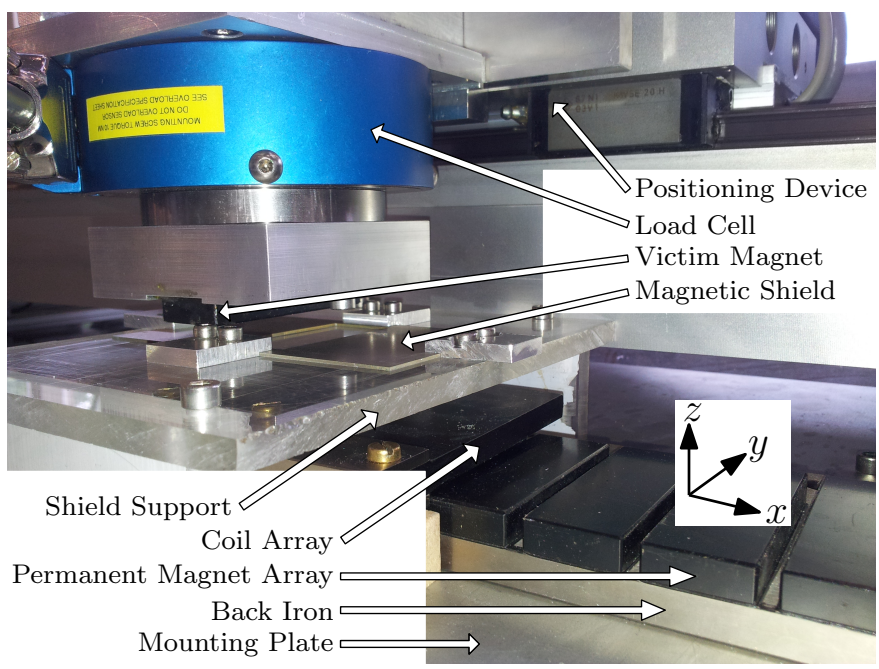

Fig. 5. The measurement setup used to verify the models.

If the $y$-dimension of the victim magnet is smaller than the $y$-dimension of the total model $Y_{t o t}$, a superposition of a 2-D model with the victim magnet and a 2-D model without the victim magnet is used. Obviously, the calculated force will be zero in the 2-D model without the victim magnet.

\section{Measurement Setup}

The findings of the superposition of 2-D models are validated by measurements performed on a measurement setup. For this measurement setup, multiple shields are manufactured, such that the variation on only one of the $y$-dimensions (either $Y_{\text {shield }}$ or $\left.Y_{\text {hole }}\right)$ at a time is possible. The used measurement setup is shown in Fig. 5, where the victim magnet is mounted to a positioning device by a 6-DOF JR3 load cell [13]. This allows for an accurate positioning of the victim magnet and the measurement of the forces experienced by this victim magnet.

On a non-magnetic mounting plate, the magnet array (i.e. half of the stator of a Tecnotion UXX ironless series motor [8]) is mounted. Above the stator a triplet of coils (from the same series) is located. The star-connected coil triplet is excited by a DC current source. The current density in the first coil is $J=5 \cdot 10^{6}\left(\mathrm{~A} / \mathrm{m}^{2}\right)$. The magnetic shield is placed on a nonmagnetic table, with the center of the shield (i.e. the center of the hole) located at $x=105(\mathrm{~mm})$ as used in the semianalytical 2-D models and the numerical 3-D models.

\section{COMPARISON OF SUPERIMPOSED 2-D MODELS WITH MEASUREMENTS AND 3-D FINITE ELEMENT ANALYSIS}

In this section a comparison between the superimposed 2-D semi-analytical models, 3-D finite element models and measurements is made. The measurements are performed on the setup described in Section V. Furthermore, a 3-D Finite Element Analysis (FEA) package, FLUX3D [14], is used to create a 3-D FEA model of the topology shown in Fig. 1. In the FEA model, the $y$-dimension of each individual model part is taken into account, while all material properties and all $x$ and $z$-dimensions are taken equal to the 2-D models.
The validity of the semi-analytical model for 3-D configurations is investigated on three different variations. First of all, the variation in the $y$-dimension of the victim magnet $\left(Y_{m a g}\right)$ is taken into account. Afterwards, the $y$-dimension of the shield $\left(Y_{\text {shield }}\right)$ is varied. The shield contains a slit throughout the full $y$-dimension of the model with a size of $X_{\text {hole }}=25(\mathrm{~mm})$ in the $x$-direction ( $X_{\text {hole }}$ is indicated in Fig. 4(c)). Finally, the $y$-dimension of the hole in the shield $\left(Y_{\text {hole }}\right)$ is varied, while the shield itself spreads the full $y$-dimension of the model, $Y_{\text {shield }}=Y_{\text {tot }}=70(\mathrm{~mm})$.

\section{A. Variation of the magnet size, $Y_{\text {mag }}$, in 3-D FEA}

For the first comparison, the 3-D FEA model and the 2-D semi-analytical model are compared for a variation in the $y$ dimension of the victim magnet, $Y_{\text {mag }}$, which is indicated in Fig. 1. In the 3-D FEA model, the $y$-dimensions of the shield is fixed to $Y_{\text {shield }}=70(\mathrm{~mm})$ and the shield contains a $X_{\text {hole }}=25(\mathrm{~mm})$ wide slit throughout the whole shield. Independent of its size, the victim magnet is centered in the $y$-direction.

Since the semi-analytical model is a 2-D model, it is not possible to individually adapt the $y$-dimension of the victim magnet. Therefore, the $y$-dimension of the total semi-analytical model is adapted. A 2-D semi-analytical model, where a shield with the hole is located underneath the victim magnet, with $Y_{\text {model }}=Y_{\text {mag }}$ is used (this model is illustrated in Fig. 4(c)). In principle, the results of a second 2-D model, a model without a victim magnet, is superimposed, however, the force obtained with that model is obviously zero. The total force calculated by the semi-analytical models is now calculated by

$$
\vec{F}_{\text {ANA }}=Y_{\text {mag }} \vec{F}_{\text {hole }}+\underbrace{\overline{\left(Y_{\text {tot }}-Y_{\text {mag }}\right)}{\overrightarrow{F_{\text {nomag }}}}}_{=0}
$$

where $Y_{\text {tot }}=70(\mathrm{~mm})$ is the $y$-dimension of the total setup (i.e. equal to the $y$-dimension of the magnet array). To illustrate the difference between the 2-D semi-analytical model and the 3-D FEA model used, an illustration of the used models is given for $Y_{\text {mag }}=10(\mathrm{~mm})$ in Fig. 6(a) and 6(b), respectively. The obtained forces from the FEA and ANA model are given in Fig. 6(c).

The results in Fig. 6(c) show that, especially in the $z$ direction, the forces are predicted very accurate. For $Y_{m a g}=$ $70(\mathrm{~mm})$, the difference found is less than $4(\%)$. For a small $y$-dimension of the magnet more deviation is found, due to the fact that the magnetic field of the victim magnet also couples to the parts of the shield that are not directly underneath, this 3-D effect is elaborated in Section VIII. Furthermore, the forces in the $x$-direction are not accurate (up to 20 (\%) deviation), this is due to the mode-matching method applied. The nonphysical boundaries assumed in the mode-matching algorithm cause a deviation in the magnetic flux density calculated by the semi-analytical model [7], which is especially found in $F_{x}$. Concluding from Fig. 6(c), the scaling of the 2-D model based on $Y_{m a g}$ (see (5)) gives an accurate prediction of the forces for this variation. 


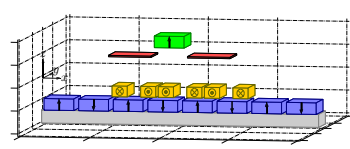

(a)

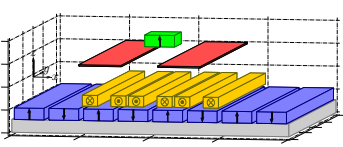

(b)

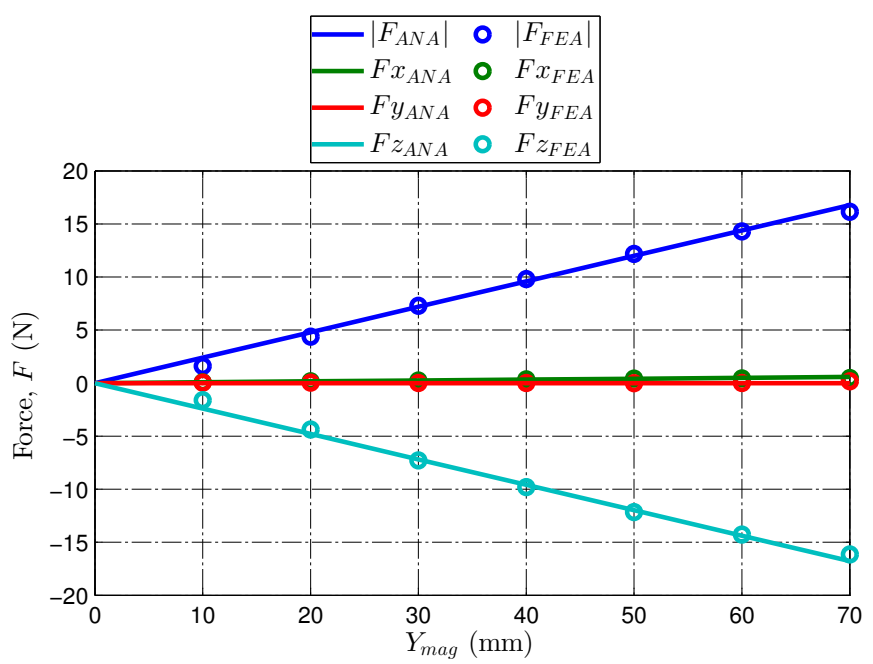

(c)

Fig. 6. The modeling results for a variation of the $y$-dimension of the victim magnet $\left(Y_{\text {mag }}\right)$, with $Y_{\text {tot }}=Y_{\text {shield }}=Y_{\text {hole }}=70(\mathrm{~mm})$. (a) The 2-D semi-analytical model used, (b) the 3-D configuration modeled in FEA and (c) the forces obtained from the models.

\section{B. Variation of the shield size, $Y_{\text {shield }}$}

The second variation used to validate the superposition of 2-D models for a 3-D topology is a variation in the $y$ dimension of the shield. The victim magnet has the full $y$-dimension of the model $Y_{\text {mag }}=70(\mathrm{~mm})$. A slit of $X_{\text {hole }}=25(\mathrm{~mm})$ wide is throughout the whole shield in the $y$-dimension. An impression of the configuration modeled in the 3-D FEA model is given in Fig. 7(b).

For the semi-analytical model, scaling the calculated force with the $y$-dimension of the shield is not sufficient. By reducing $Y_{\text {shield }}$, more of the magnet plate underneath is revealed to the victim magnet. Therefore, the expected force obtained with the semi-analytical model is a superposition of two separate semi-analytical models. One model with a shield and the $y$ dimension of the model equal to the shield dimension (this model is illustrated in Fig. 4(c)). The second model is a model without a shield, as illustrated in Fig. 4(a), with a $y$-dimension equal to the remaining part of the victim magnet which is not covered by the shield. The total force on the victim magnet is then obtained by

$$
\vec{F}_{\text {ANA }}=Y_{\text {shield }} \vec{F}_{\text {hole }}+Y_{\text {no shield }} \vec{F}_{\text {no shield }}
$$

where $Y_{\text {shield }}$ is the $y$-dimensions of the shield, $Y_{\text {no shield }}=$ $Y_{\text {mag }}-Y_{\text {shield }}$ is the $y$-dimension of the victim magnet which is not covered by the shield, and where $\vec{F}_{\text {hole }}$ and $\vec{F}_{\text {no shield }}$ are the forces obtained from the semi-analytical model when the shield with the hole is present and when no shield is present, given in Fig. 4(c) and 4(a), respectively. For the

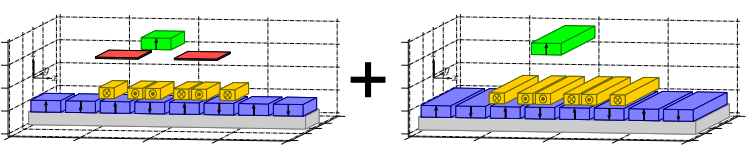

(a)

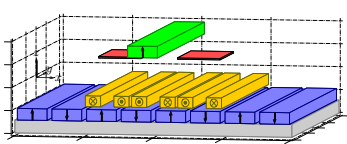

(b)

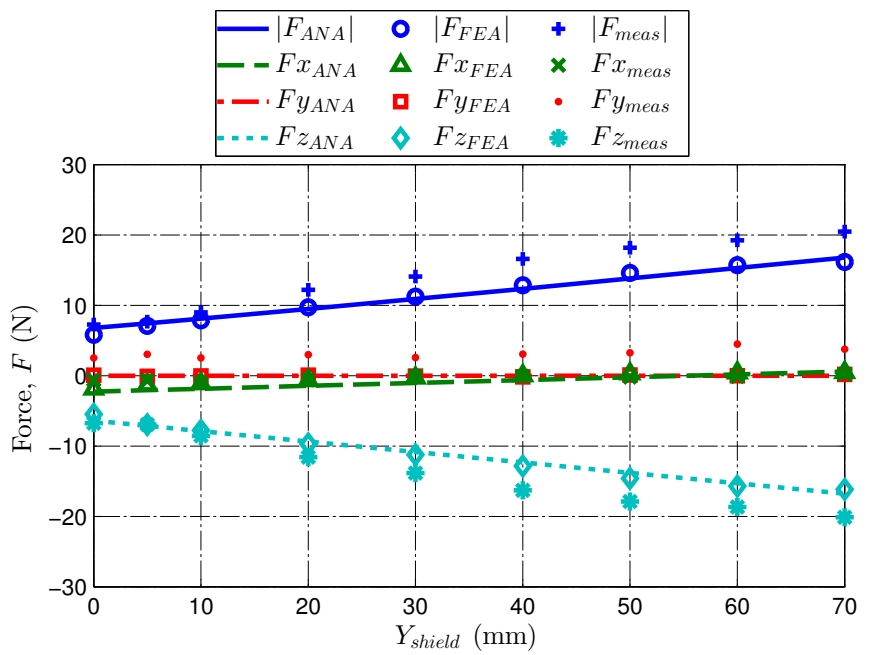

(c)

Fig. 7. The models and results for the variation of the $y$-dimension of the shield ( $Y_{\text {shield }}$ ), with $Y_{\text {tot }}=Y_{\text {mag }}=Y_{\text {hole }}=70(\mathrm{~mm})$. (a) The superposition of the two 2-D semi-analytical models used, (b) the 3-D configuration as modeled in FEA and (c) the actual forces obtained from the models (ANA is the superposition of 2-D models, FEA is the 3-D Finite element model) and the measurements (meas).

situation with $Y_{\text {shield }}=20(\mathrm{~mm})$, an illustration of the superposition of these two semi-analytical models is given in Fig. 7(a), while the obtained forces from the FEA and ANA model are given in Fig. 7(c). Measurements for the victim magnet centered above the hole in the shield, for the different shield sizes are included as well.

From the results shown in Fig. 7(c), it is clear that the tendencies in the force variations are predicted reasonably accurate by the superposition of the 2-D semi-analytical models. From the results is found that the predicted forces in the $z$ direction by the 2-D models are within 17 (\%) of the 3-D FEA models, while $\leq 22(\%)$ difference with the measurements remains. For $Y_{\text {shield }}=0(\mathrm{~mm})$ and $Y_{\text {shield }}=70(\mathrm{~mm})$, the remaining difference between the 3-D FEA and the 2-D ANA, $17(\%)$ and $4(\%)$, respectively, is mainly caused by the 3-D end-effects. Another observation is the slightly non-linear behavior of especially the force in the $z$-direction found from the 3-D FEA model. The deviation of the predicted linear behavior by the superposition of the 2-D semi-analytical models is caused by a 3-D effect, where the magnetic field is drawn into the shield from a larger part of the victim magnet than only the part that is exactly above the shield. These 3-D 
effects are elaborated on in the discussion (Section VIII).

Furthermore, from both the 2-D semi-analytical and the 3-D FEA models, no force is expected in the $y$-direction $\left(F y_{A N A}=F y_{F E A}=0(\mathrm{~N})\right)$, while the measurements clearly show a residual force in the $y$-direction. This residual force is caused by features of the test setup that are not (correctly) modeled, such as inaccurate placement of the victim magnet above the magnet array in the $y$-direction, inaccurate placement of the shield in the $y$-direction, a longer back-iron in the $y$-direction than the permanent magnets and possible saturation in the shield (as elaborated on in Section VIII).

Since the tendencies and the order of magnitude predicted by the semi-analytical models are correct, it is found that the superposition of the 2-D semi-analytical models scaled according to (6) for a variation of the $y$-dimension of the shield holds.

\section{Variation of the hole size, $Y_{\text {hole }}$}

The third validation is performed on a variation of the $y$ dimension of the hole in the shield. A victim magnet and a shield, both with the full $y$-dimension of the model, are present, $Y_{\text {mag }}=Y_{\text {shield }}=Y_{\text {tot }}=70(\mathrm{~mm})$. Centered in the shield there is a rectangular hole which always has an $x$-dimension of $X_{\text {hole }}=25(\mathrm{~mm})$ and has a variable $y$ dimension. The 3-D configuration as modeled in FEA for $Y_{\text {hole }}=35(\mathrm{~mm})$ is given in Fig. 8(b).

For this variation, a superposition of two semi-analytical models is necessary as well. When the $y$-dimension of the hole in the shield is reduced, more of the victim magnet is covered by a full shield of a single piece. Therefore, the two models used are, a model with the shield containing the hole / slit (illustrated in Fig. 4(c)), and a model with a full shield (containing no hole as illustrated in Fig. 4(b)). By superimposing the results of these two semi-analytical models scaled by the $y$-dimension of the hole, the force of the analytical model is given by

$$
\vec{F}_{\text {ANA }}=Y_{\text {hole }} \vec{F}_{\text {hole }}+\left(Y_{\text {mag }}-Y_{\text {hole }}\right) \vec{F}_{\text {shield }}
$$

where $Y_{\text {hole }}$ and $Y_{\text {mag }}=70(\mathrm{~mm})$ are the $y$-dimensions of the hole and the victim magnet, respectively, and where $\vec{F}_{\text {hole }}$ and $\vec{F}_{\text {shield }}$ are the forces obtained from the semi-analytical model when the shield with the hole is present and when the full shield is present, respectively. The obtained forces from the FEA and ANA model and the measurements are given in Fig. 8(c).

The 2-D modeling results, shown in Fig. 8(c), for the force in the $z$-direction are deviating slightly with the 3-D FEA results, up to $13(\%)$ difference is found for some values of $Y_{\text {hole }}$. A closer observation shows that the value for $Y_{\text {hole }}=0(\mathrm{~mm})$ and $Y_{\text {hole }}=70(\mathrm{~mm})$ predicted by the 2-D models are accurate, less than $4(\%)$ difference. This leads to the conclusion that the deviation between the semianalytical and the FEA models for the intermediate values of $Y_{\text {hole }}$ is caused by 3-D effects. The magnetic field from the part of the victim magnet that is located above the hole is drawn into the shield in the $y$-direction as well. This

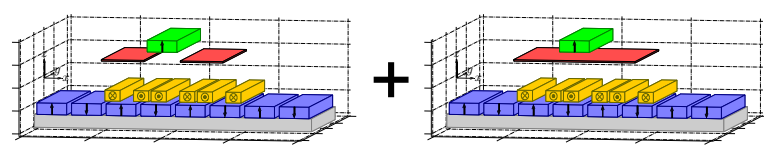

(a)

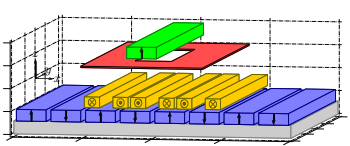

(b)

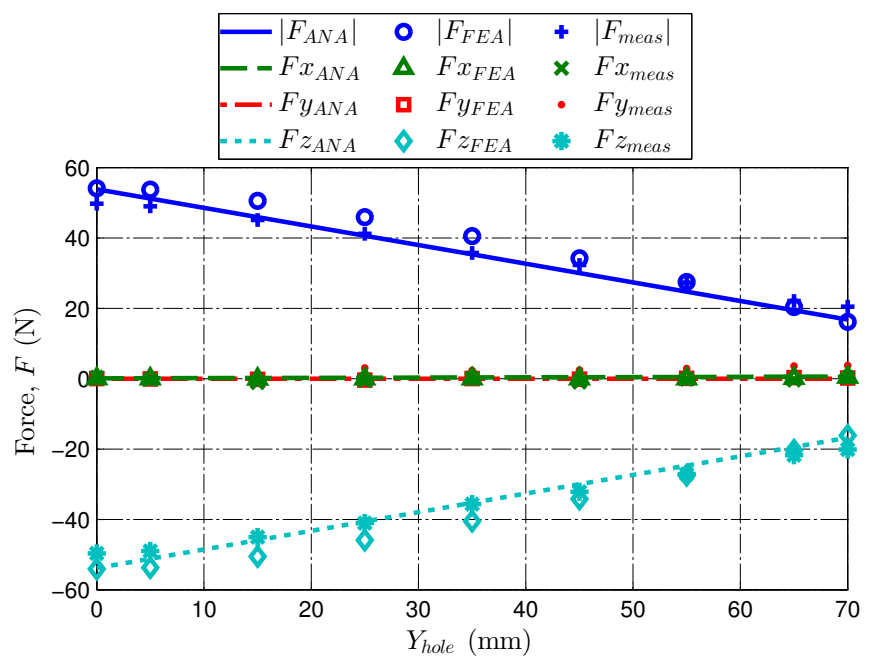

(c)

Fig. 8. The models and results for the variation of the $y$-dimension of the hole $\left(Y_{\text {hole }}\right)$, with $Y_{\text {tot }}=Y_{\text {mag }}=Y_{\text {shield }}=70(\mathrm{~mm})$. (a) The superposition of the two 2-D semi-analytical models used, (b) the 3-D configuration modeled in FEA and (c) the actual forces obtained from the models (ANA is the superposition of 2-D models, FEA is the 3-D Finite element model) and the measurements (meas).

is not incorporated in the 2-D models since these assume that magnetic flux density in the $y$-direction will be zero throughout the full model. This effect is elaborated upon in Section VIII.

Another interesting observation is the slight difference in the slope of the measurements and the models. Clearly some of the effects in the measurements are not captured by either of the modeling techniques. The most probable causes of the differences between the models and the measurements are saturation of the magnetic shield (as will be elaborated on in Section VIII), and that the back-iron is extending behind the permanent magnets of the magnet plate in the measurement setup.

Based on the results shown in Fig. 8(c), it is clear that superimposing the results of the 2-D semi-analytical models scaled according to their dimensions (as given in (7)) gives a good prediction of the actual forces for the variation of the hole size $Y_{\text {hole }}$. 


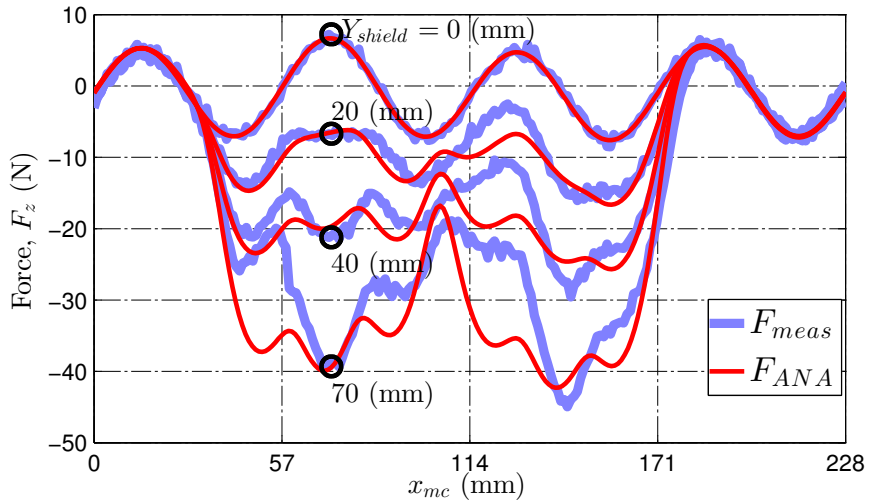

Fig. 9. The measured $F_{\text {meas }}$ and analytically calculated force $F_{A N A}$ in the $z$-direction on the victim magnet for a movement of the magnet in the $x$-direction over the full periodicity for different $y$-dimensions of the shield, $Y_{\text {shield }}$.

\section{COMPARISON OF SUPERIMPOSED 2-D MODELS WITH MEASURED 3-D SITUATIONS}

For a more thorough comparison between the measurements and the 2-D models, the victim magnet has been moved in the $x$-direction over the full period, instead of only measuring one individual point.

\section{A. Measurements on a moving victim magnet for different shield sizes, $Y_{\text {shield }}$}

The results for a variation of the shield size, as calculated by the semi-analytical model and as obtained with the measurements, for the movement of the victim magnet over the full periodicity is shown in Fig. 9, where $Y_{\text {tot }}=Y_{\text {hole }}=$ $Y_{\text {mag }}=70(\mathrm{~mm})$. For four different shield sizes $\left(Y_{\text {shield }}\right)$, the measurements and the superimposed 2-D modeling results are given, where $x_{m c}$ is the $x$-position of the center of the victim magnet.

The figure clearly shows, that for the situation of $Y_{\text {shield }}=0(\mathrm{~mm})$, the semi-analytical 2-D model and the measurements are in very good agreement. Based on the figure, both the 2-D models predict, and the measurements show a large attraction force towards the shield for an increasing shield size. The attraction force is acting in the negative $z$ direction. Furthermore, the sinusoidal force behavior acting from the permanent magnet plate on the victim magnet (see $\left.Y_{\text {shield }}=0(\mathrm{~mm})\right)$, is strongly reduced by the shield (compare $Y_{\text {shield }}=0(\mathrm{~mm})$ and $\left.Y_{\text {shield }}=20(\mathrm{~mm})\right)$, if the victim magnet is located above the shield.

The forces for $Y_{\text {shield }}=70(\mathrm{~mm})$ are not accurately predicted by the 2-D semi-analytical model. The measured situation is equal to the modeled situation in 2-D , since all parts of the topology have an equal $y$-dimension. The remaining difference should therefore be caused by a nonmodeled feature, which can be either saturation or a 3-D effect (i.e. end-effects). Since the comparison with 3-D FEA for a variation of the shield size only showed a deviation of approximately 17 (\%) (see Fig. 7(c)), it is likely that the differences between the 2-D models and the measurements

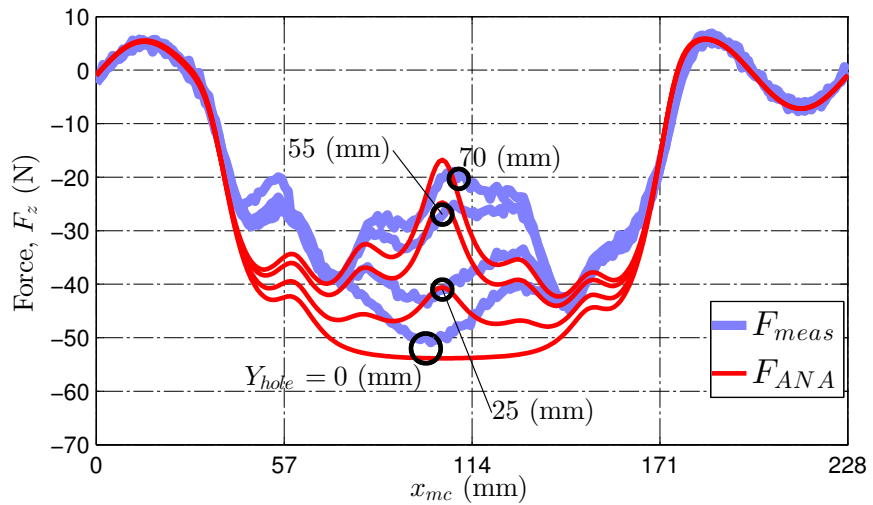

Fig. 10. The measured $F_{m e a s}$ and analytically calculated force $F_{A N A}$ in the $z$-direction on the victim magnet for a movement of the magnet in the $x$-direction over the full periodicity, for different $y$-dimensions of the hole, $Y_{\text {hole. }}$.

are mainly caused by saturation, which is further discussed in Section VIII.

Based on Fig. 9, it is found that the assumed superposition of a 2-D model without a shield and a 2-D model with a shield containing a hole, scaled by their respective $y$-dimension (6) gives a good initial prediction of the forces in this variation.

\section{B. Measurements on a moving victim magnet for different hole} size, $Y_{\text {hole }}$

For the variation of the $y$-dimension of the hole, the results of the semi-analytical model and the measurements are given in Fig. 10 for the movement of the victim magnet over the full periodicity, where $Y_{\text {tot }}=Y_{\text {shield }}=Y_{\text {mag }}=70(\mathrm{~mm})$. For four different hole sizes $\left(Y_{\text {hole }}\right)$, the measurements and the superimposed 2-D modeling results are given.

In the figure, it is found that the difference between the superimposed semi-analytical 2-D models and the measurements is larger as in the previous variation. For the situation with $Y_{\text {hole }}=0(\mathrm{~mm})$, the geometry of the measured situation and the 2-D model with a full shield are equal. Therefore, the difference found between the obtained force for the model and measurement are caused by either saturation of the shield or by the 3-D end-effects. Based on the comparison of the 2-D semi-analytical models and the 3-D FEA models, the difference caused by 3-D effects are very limited $(\leq 1(\%)$ for $Y_{\text {hole }}=0(\mathrm{~mm})$, and $\leq 4(\%)$ for $Y_{\text {hole }}=70(\mathrm{~mm})$ ). Therefore, saturation is the most probable cause of the differences for $Y_{\text {hole }}=0(\mathrm{~mm})$ and $Y_{\text {hole }}=70(\mathrm{~mm})$.

The tendency of reducing the attraction force when the victim magnet is centered above the hole for an increasing hole size is captured well by the 2-D modeling. It is found that superimposing the 2-D models for analysis of a 3-D situation gives a reasonable indication of the forces present. However, for the variation of $Y_{\text {hole }}$, both saturation and the 3-D effects are not negligible as will be discussed in the next section.

\section{DISCUSSION}

With the superposition of 2-D models, the geometrical differences in the $y$-direction are included, however, the fact 
that the magnetic field will have a three-dimensional nature, and therefore, has a magnetic field in the $y$-direction is still neglected with this approach, which is discussed in the first part of this section. Furthermore, the second part of this section will elaborate on the deviations originating due to the saturation of the shield, while the third part considers the computational efforts.

\section{A. 3-D effects}

As was indicated in the previous sections, the 3-D effects in the measurements and the 3-D FEA models might cause the differences with the 2-D models. One of the well known 3-D effects is the end-effect. The end-effect for a very simple geometry, a (victim) magnet and a (full) shield, with comparable dimensions and distance, is illustrated in Fig. 11(a). In the figure, the geometry is observed in a cross-section in the $y z$-plane in the center of the magnet, and therefore, shows the non-modeled dimension of the 2-D models. The thin dotted lines are the assumed flux lines for the 2-D model, which only exist in the part where the 2-D model holds, while the thick solid lines, the actual flux lines in the 3-D geometry, are spreading across a far bigger $y$-dimension than the 2-D lines does. The 3-D end-effect for $Y_{\text {mag }}=Y_{\text {shield }}=70(\mathrm{~mm})$, is up to $4(\%)$ as indicated by the comparison between the 2-D semi-analytical models and the 3-D FEA model in Fig. 7(c).

In Fig. 11(b), the 3-D effects are illustrated for the situations where the $y$-dimension of the shield is varied, $Y_{\text {shield }}$. As can be seen, this situation shows, besides the 3-D end-effects (Fig. 11(a)), also a bending of the flux-lines towards the shield. In the 2-D models only vertical magnetic field lines are assumed in this situation (as indicated by the thin dotted lines), while the solid flux lines of the 3-D situation give a bending of the magnetic field towards the shield. This results in an attraction force of the victim magnet towards the shield from a larger part of the victim magnet than the part of the victim magnet that is located directly above the shield, as is assumed in a 2-D model. Based on the comparison between the 2-D semi-analytical models and the 3-D FEA model (see Fig. 7(c)), this 3-D effect could cause up to $17(\%)$ difference, for the topology concerned in this paper.

In Fig. 11(c) another 3-D effect is illustrated. For a variation of the $y$-dimension of the hole, the shield is closed underneath the ends of the victim magnet. This situation is illustrated in the figure, where it is shown that the flux lines are bending towards the shield from the middle of the magnet as well. In a 2-D model, it is assumed that only flux lines of the parts of the magnet that are located above the shield are coupled through the shield (this is illustrated by the thin dotted lines). Based on the comparison between the 2-D semi-analytical models and the 3-D FEA model (see Fig. 8(c)) for the topology considered, this 3-D effect could cause up to $13(\%)$ difference.

To make the 3-D effects in this topology even more visible, the magnetic flux density in a $x y$-plane inside the shield is given in Fig. 12. The magnetic flux density obtained with the superposition of the 2-D semi-analytical models (see Fig. 12(a)) and from the 3-D FEA model (see Fig. 12(b)),

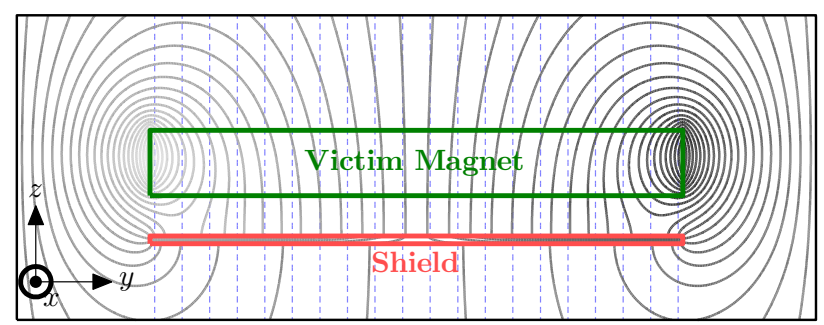

(a)

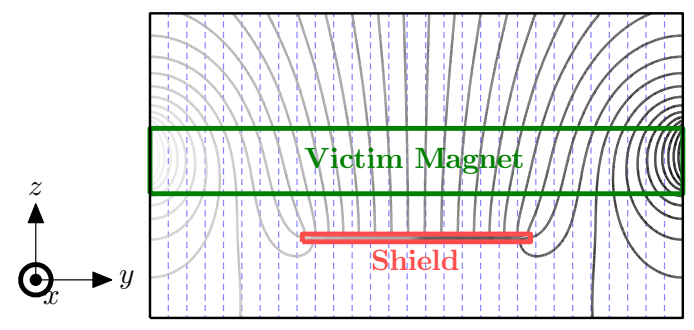

(b)

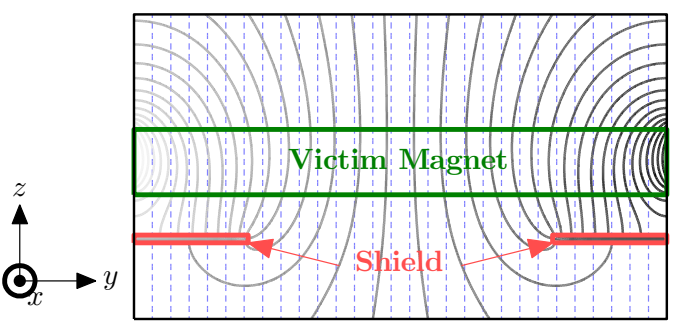

(c)

Fig. 11. An illustration of the flux lines in the $y z$-plane, this indicates the 3-D effects present in the 3-D model. (a) The end-effect, (b) concentration of flux towards the shield (for variation of $Y_{\text {shield }}$ ) and (c) concentration of flux towards the shield (for variation of $Y_{\text {hole }}$ ).

for $Y_{\text {tot }}=Y_{\text {mag }}=Y_{\text {shield }}=70(\mathrm{~mm})$ and $Y_{\text {hole }}=45(\mathrm{~mm})$ is shown. Comparing these figures, it is clear that the magnetic flux density is differently distributed when superimposing 2-D models than in the actual 3-D situation. However, comparing the magnetic flux density distribution of Fig. 12(b) at $y=0(\mathrm{~mm})$ and $y=70(\mathrm{~mm})$ with Fig. 12(a) at $y=0(\mathrm{~mm})$ and $y=70(\mathrm{~mm})$, a very good agreement is found.

\section{B. Saturation}

Besides the 3-D effects, saturation might be of influence in the measurements as well. For the 3-D finite element models, a linear material is assumed as is done in the analytical models. However, in the measurements, the material used (S235) is non-linear. Based on the superposition of the 2-D analytical models, the expected magnetic flux density is given in Fig. 12(a). The figure clearly shows that a large part of the magnetic shielding material is approaching or above $2(\mathrm{~T})$. This indicates that those parts of the shield are saturated. (The peak value of the magnetic flux density found inside the shield is equal to $3.2(\mathrm{~T})$.) The amount and location of saturation 


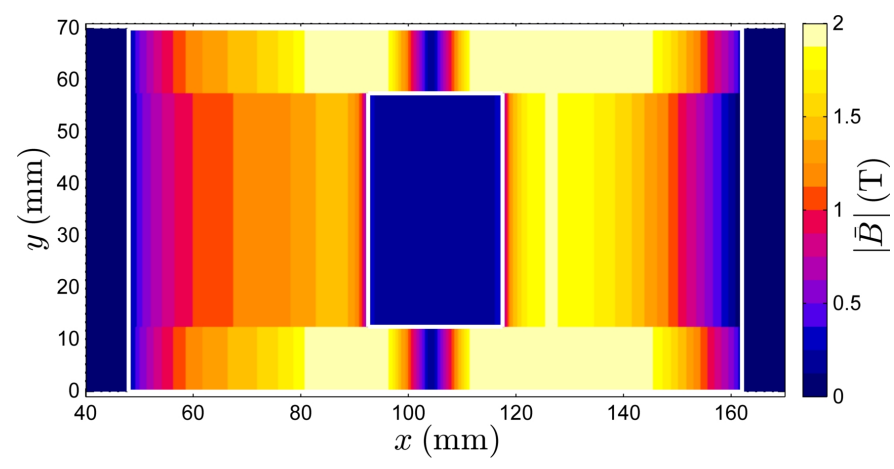

(a)

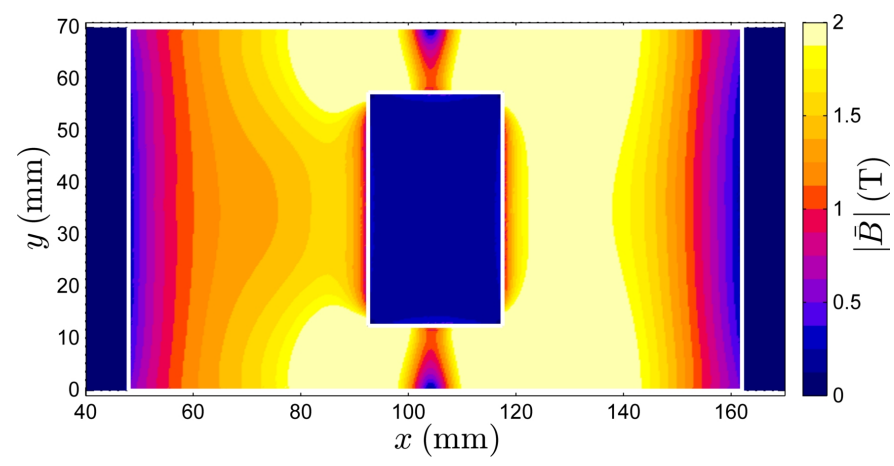

(b)

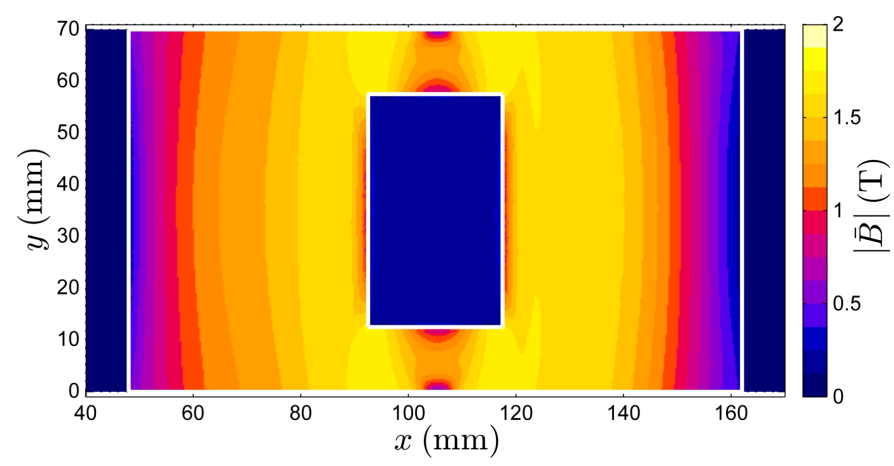

(c)

Fig. 12. The magnetic flux density in the $x y$-plane in the middle of the shield for (a) the superposition of 2-D semi-analytical models and (b) the 3-D FEA model with linear shielding material and (c) the 3-D FEA mode with non-linear material in the shield, where the white solid line indicates the shield size for both models.

are strongly dependent on the position of the victim magnet.

To estimate the influence of the saturation in the current situation, a 3-D FEA model is made with non-linear material properties. For this model, the BH-curve of the material is measured, and the resulting values are used for a splineinterpolation in the 3-D FEA software. Based on this nonlinear 3-D FEA model, the magnetic flux density inside the magnetic shield is given by Fig. 12(c). As it can clearly be seen when comparing the results with Fig. 12(b), saturation is present in the measured situation. For the situation where $Y_{\text {hole }}=45(\mathrm{~mm})$, the difference in the forces as predicted by the linear and the non-linear FEA are up to $25(\%)$. Therefore, the saturation is significantly influencing the force results in the measurements.

The amount of saturation in the geometry can be lowered by increasing the distance between the individual modeling parts. However, increasing the distance in the topology will increase the 3-D effects since the magnetic field is more 2-D for a topology where only small distances are present. For the accuracy of the modeling of a 3-D geometry with a 2-D modeling method with linear materials, the distances between the individual parts are a trade-off between saturation and 3-D effects.

\section{Computational efforts}

During the initial design process of a magnetic shield, a fast prediction of the influence of this magnetic shield is necessary. The computational time necessary for the linear 3-D FEA models used is more than 1 hour for a single point of calculation (one position of the victim magnet, $x_{m c}$, one victim magnet dimension, $Y_{\text {mag }}$, one shield dimension, $Y_{\text {shield }}$ and one hole dimension, $Y_{\text {hole }}$ ). When using a 3-D FEA with non-linear materials, the computational time is even larger. For the 2-D semi-analytical model, however, the same single point of calculation is finished in less than 5 seconds. Therefore, from computational point of view, the superposition of the 2-D semi-analytical models is very valuable.

\section{CONCLUSiON}

To predict the influence of the application of magnetic shielding, this paper employed the superposition of multiple 2-D models to describe the 3-D shielding configuration. By subdividing the $3-\mathrm{D}$ structure into invariant parts in the $y$ dimension, multiple 2-D semi-analytical models have been obtained. The forces per unit depth calculated with these 2-D models are multiplied by the depth of the associated invariant part and summed to obtain the value for the 3-D model.

From the comparison of the results of the 2-D semianalytical models with 3-D FEA models is concluded that the semi-analytical models give a good prediction of both the global tendencies and the order of magnitude of the forces involved. For the situations where all $y$-dimensions of the 3-D FEA model are equal the difference between the obtained forces are below $4(\%)$. Based on the measurements, the 2-D semi-analytical models show more deviation than expected based on the 3-D FEA models, which is explained by the saturation of the shielding material. However, for all measurements, the 2-D models predicted the right tendencies and, therefore, an accurate initial prediction of the shielding effect is found with the 2-D models. Especially from computational point of view, the superposition of semi-analytical 2-D models is very valuable during 3-D shielding design, even though the actual situation is three-dimensional.

\section{REFERENCES}

[1] M. Andriollo, G. Bettanini, and A. Tortella, "Design procedure of a small-size axial flux motor with halbach-type permanent magnet rotor and smc cores," in Electric Machines Drives Conference (IEMDC), 2013 IEEE International, 2013, pp. 775-780. 
[2] B. Funieru and A. Binder, "Simulation of electrical machines end effects with reduced length $3 \mathrm{~d}$ fem models," in Electrical Machines (ICEM), 2012 XXth International Conference on, 2012, pp. 1430-1436.

[3] J. J. Cathey, Electric Machines: Analysis and Design Applying MATLAB, 1st ed. McGraw-Hill Higher Education, 2000.

[4] V. Ruuskanen, J. Nerg, and J. Pyrhonen, "Effect of lamination stack ends and radial cooling channels on no-load voltage and inductances of permanent-magnet synchronous machines," Magnetics, IEEE Transactions on, vol. 47, no. 11, pp. 4643-4649, 2011.

[5] Z. Zhang, N. Cheung, K. Cheng, X. Xue, and J. Lin, "Longitudinal and transversal end-effects analysis of linear switched reluctance motor," Magnetics, IEEE Transactions on, vol. 47, no. 10, pp. 3979-3982, 2011.

[6] J. Gieras, Linear Induction Drives, ser. Monographs in electrical and electronic engineering. Clarendon Press, 1994.

[7] K. J. W. Pluk, J. W. Jansen, and E. A. Lomonova, "Magnetic shielding for coreless linear permanent magnet motors," Applied Mechanics and Materials, vol. 416, pp. 45-52, 2013.

[8] Tecnotion UXX Series Ironless, Tecnotion BV.
[9] B. L. J. Gysen, K. J. Meessen, J. J. H. Paulides, and E. A. Lomonova, "General formulation of the electromagnetic field distribution in machines and devices using fourier analysis," Magnetics, IEEE Transactions on, vol. 46, no. 1 , pp. $39-52$, Jan 2010

[10] K. J. W. Pluk, G. de Gersem, J. W. Jansen, and E. A. Lomonova, "Field calculations for magnetic shielding: Fourier modeling extended with mode-matching technique applied on a shield with finite dimensions," Magnetics, IEEE Transactions on, vol. 49, no. 5, pp. 1593-1596, 2013.

[11] K. J. W. Pluk, G. De Gersem, J. W. Jansen, and E. A. Lomonova, "Fourier modeling of magnetic shields with linear permeable material and finite dimensions," Magnetics, IEEE Transactions on, vol. 49, no. 7, pp. 4160-4163, 2013.

[12] K. J. W. Pluk, J. W. Jansen, and E. A. Lomonova, "Force measurements on a shielded coreless linear permanent magnet motor," Magnetics, IEEE Transactions on, Accepted for publication, 2014.

[13] JR3 Model No. 45E15A4-I63-S 100N10 Multi-Axis Force-Torque Sensor, JR3, Inc., Woodland, CA 95776.

[14] Cedrat, FLUX 11.2.0, User's guide, Meylan, France, 2013. 